\title{
Spectrum Trading for Efficient Spectrum Utilization
}

\author{
Cong Xiong ${ }^{1, *}$, Geoffrey $\mathrm{Ye} \mathrm{Li}^{1}, \mathrm{Lu} \mathrm{Lu}^{1}$, Daquan Feng ${ }^{1,2}, \mathrm{Zhi} \mathrm{Ding}^{3}$, and Helena Mitchell ${ }^{4}$ \\ ${ }^{1}$ School of Electrical and Computer Engineering, Georgia Institute of Technology, Atlanta, GA, USA \\ ${ }^{2}$ National Key Lab on Commun., University of Electronic Science and Technology of China, Chengdu, China \\ ${ }^{3}$ School of Electrical and Computer Engineering, University of California, Davis, CA, USA \\ ${ }^{4}$ Center for Advanced Communications Policy, Georgia Institute of Technology, Atlanta, GA, USA
}

\section{Abstract}

The conventional command and control based spectrum management has led to substantial underutilization of some spectrum bands while severely crowding others due to the uneven and dynamic needs that vary over time and at different locations. Spectrum trading has emerged as a promising management approach to substantially improve spectrum utilization and user experience in wireless communications by taking advantage of market-based mechanisms. This article presents an overview of spectrum trading, including the fundamental characteristics of spectrum trading markets, the state-of-the-art techniques for modeling and resolving various spectrum trading issues, and trading based dynamic spectrum sharing and access. Moreover, some open issues in spectrum trading are identified for future research in this area.

Received on 20 September 2013; accepted on 29 January 2014; published on 11 April 2014

Keywords: spectrum market, spectrum trading, dynamic spectrum sharing and access

Copyright (C) 2014 Cong Xiong et al., licensed to ICST. This is an open access article distributed under the terms of the Creative Commons Attribution license (http://creativecommons . org/licenses/by/3.0/), which permits unlimited use, distribution and reproduction in any medium so long as the original work is properly cited.

doi:10.4108/ws.1.1.e1

\section{Introduction}

Traditionally, radio spectrum is a highly regulated commodity whose management and coordination involve a central government entity, such as the Federal Communications Commission (FCC) in the United States. Such centralized command and control based spectrum assignment mechanism typically predicts and predetermines static bands for their respective specific usage without accounting for the dynamic nature of radio frequency (RF) spectrum quality and the service requirements. As a result, most preassigned spectrum bands can be substantially underutilized while many highly popular civilian bands are increasingly overcrowded, particularly due to the recent boom of many wireless applications [1-4]. For example, TV broadcast bands are routinely underutilized while cellular mobile bands are becoming overly crowded. In brief, such a centralized spectrum planning is simplistic, often leading to poor spectral efficiency, and is difficult to attain the full social and economic values for the spectrum.

${ }^{*}$ Corresponding author. Email: xiongcong@gatech.edu
Auction is a traditional market mechanism used by regulatory agencies to release the unallocated spectrum to bidders. Once the initial auction is over, the winners are licensed to use the auctioned spectrum bands. Generally, they cannot further trade their acquired spectrum on the market $[5,6]$. To fully involve economic incentives in spectrum trading to achieve high spectrum utilization efficiency, subsequent markets that permit spectrum licensees to flexibly choose among capital investment, spectrum utilization, or profit trading on a dynamic basis are desired beyond the initial auction and assignment [5-8].

Unlike many popular approaches to dynamic spectrum assignment, with focus either on finding vacant spectrum for unlicensed systems or on noncooperative sharing between licensed and unlicensed users, spectrum trading is a more proactive and open form of cooperative spectrum sharing that involves temporary or long-term spectrum license transfers for economic reasons [5-8]. In essence, spectrum trading presents various spectrum seekers an opportunity to take over spectrum bands and deliver better values, thereby facilitating the establishment of dynamic incentive-driven and competitive wireless communication markets. 
Liberalized trading of spectrum licenses can potentially take better advantages of market-based economic mechanisms, leading to maximum social and economic utilities of spectrum [5-10]. Specifically, in establishing a fully competitive and cooperative wireless market, wireless service providers are expected to increase their profits by thoroughly exploiting the benefits and characteristics of different spectrum bands and appropriately allocating resources across a wide swath of multi-spectrum bands. Users will have more choices for better services at lower prices. Society can better fulfill practical RF spectrum needs, including public safety, telemedicine, and social services. Thus, spectrum trading based on market supply and demand is of growing interest to regulatory agencies, social and economic studies, as well as academic and industrial wireless research groups.

This article focuses on spectrum trading in wireless communications. We present an overview on recent development of spectrum trading market including known techniques and also point out some open challenges. The rest of this article is organized as follows. Section 2 introduces several models for spectrum trading markets. Sections 3, 4, and 5 investigate several important issues of spectrum trading, models and methods for spectrum trading, and spectrum trading based dynamic spectrum sharing and access, respectively. We also highlight some open issues in Section 6. Finally, we conclude this paper in Section 7.

\section{Spectrum Trading Markets}

The desire to use market-based mechanisms instead of traditional command and control methods for spectrum management has motivated studies on the forms and viability of future spectrum trading markets. In general, the spectrum trading market may consist of entities, such as spectrum sellers, spectrum buyers, spectrum brokers, and a regulator, as illustrated in Figure 1. Their functions and the interaction among them depend on the spectrum trading market models. In this section, we introduce three existing spectrum trading market models, including the roles of the involved entities, which can be used to analyze the potential of spectrum trading markets. These spectrum trading market models are briefly summarized in Table 1 .

\subsection{Secondary Spectrum Trading Markets}

In the traditional centrally controlled spectrum market, the assignment of newly released spectrum is usually static, lasting for a long term (e.g. tens of years) and covering a large geographic area (e.g., national-wide). However, such an approach has caused substantial underutilization of some licensed spectrum bands while badly crowding others [1].

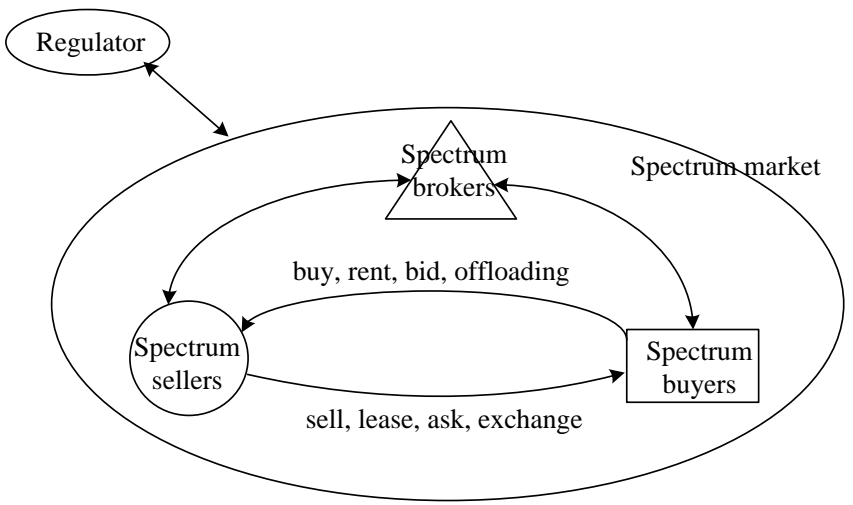

Figure 1. Structure of spectrum trading markets.

Realizing the potential power of market forces in improving the overall utilization efficiency of spectrum, governmental regulatory agencies have acknowledged the possibility for existing spectrum licensees to trade their unused spare spectrum through secondary spectrum markets under certain rules [2-4]. A secondary spectrum market allows and encourages existing spectrum licensees to trade away their rights to unused spectrum, either leasing it temporarily, or on a longer-term basis, or even outright selling their rights, to other organizations that badly need this spectrum. We believe that an effectively functioning secondary spectrum market can increase the amount of spectrum capacity available to prospective wireless service demands and new wireless technologies by substantially improving the utilization of spectrum currently assigned to less active license holders.

To facilitate successful development of secondary spectrum markets, two categories of spectrum leasing options have been proposed [2, 3]. One leasing option, namely "spectrum manager" leasing, permits entities to engage in leasing arrangements without prior governmental approval as long as the original licensee retains both de jure control of the license and de facto control over the leased spectrum. The other option, known as "de facto transfer" leasing, creates a streamlined approval process for leases that involve a transfer of the de facto control of the license.

The spectrum market based on the concept of secondary spectrum market adopts some marketdriven mechanisms for spectrum management and is a smooth evolution of the command and control based spectrum market. However, it still lacks the high-level liberalization of commodity market and involves a certain level of governmental regulation and restriction, which may hinder the efficient reallocation of spectrum. 
Table. 1. Comparison of different models for spectrum trading market

\begin{tabular}{c|l}
\hline \multicolumn{1}{c|}{ Model } & \multicolumn{1}{c}{ Features } \\
\hline Secondary spectrum trading market & $\begin{array}{l}\text { spectrum licensees trade with buyers directly } \\
\text { spectrum leasing without transfer of de jure control of license } \\
\text { low-level of market-based mechanisms, high-level of regulation }\end{array}$ \\
\hline \multirow{2}{*}{ Exchanged-based spectrum trading market } & $\begin{array}{l}\text { market maker facilitates trading as a dealer } \\
\text { spectrum leasing with and without transfer of de jure control of license } \\
\text { medium-level of market-based mechanisms, medium-level of regulation }\end{array}$ \\
\hline Two-tire spectrum trading market & $\begin{array}{l}\text { separation of spectrum ownership from provision of wireless service } \\
\text { spectrum broker manages spectrum assets } \\
\text { high-level of market-based mechanisms, low-level of regulation }\end{array}$ \\
\hline
\end{tabular}

\subsection{Exchange-based Spectrum Trading Markets}

To introduce more market-forces into spectrum trading, an exchange-based spectrum trading market is proposed in [5]. The entities participating in the exchangebased spectrum market include spectrum exchange, spectrum license holders, spectrum license requestors, spectrum regulators, and market makers. Spectrum exchange maintains facilities for spectrum trading; spectrum license holders own spectrum license and want to sell it; spectrum license requestors want to buy spectrum licenses; spectrum regulators oversee the spectrum trading market; and market makers facilitate trading and acts as a dealer that holds an inventory of spectrum. In a typical trade of the exchange-based spectrum market, the spectrum exchange collects offers to sell spectrum from spectrum license holders and offers to buy spectrum from spectrum license requestors, determines the winning bid, and transfers a spectrum usage right from its holder to its winning requestor.

Compared to the secondary spectrum trading markets, exchange-based spectrum trading markets have more liberalized market structure and may exploit more benefits from general market forces.

\subsection{Two-Tier Spectrum Trading Markets}

To fully exploit the power of market-based mechanisms, a two-tier spectrum trading market [6] is suggested, which advocates the separation of spectrum ownership from the provision of wireless services as a reasonable outcome of trading spectrum property right. The upper-tier market involves spectrum owners trading spectrum property rights similar to other commodity rights, whereas the lower-tier market consists of spot markets for service providers to acquire limitedduration rentals of spectrum assets from owners at particular locations. Spectrum brokers managing spectrum assets at particular locations play the role of middlemen for spectrum owners of the upper tier and service providers of the lower tier. Note that the two market tiers could run at different time-scales: spectrum assets at the upper tier might be traded on a long-term scale, whereas rented spectrum assets at the lower tier could be negotiated over short-term time scales.

The two-tier spectrum market potentially enables relatively low trading/transcation and maintenance costs for wireless service providers, especially for small and short-term acquisition. The entry barriers for wireless service market may also be lowered, leading to more competitive and diversified services and more efficient utilization of the entire spectrum. However, whether such high-level liberalization of the spectrum will damage some social value aspects, such as fairness, of spectrum utilization and how to avoid the possible damage need to be carefully addressed.

\section{Important Issues for Spectrum Trading}

In this section, we discuss some important issues in various spectrum trading activities, including pricing mechanism and utility functions.

\subsection{Pricing Mechanism}

In spectrum trading, price reflects the value of the spectrum bands to their sellers and buyers. Therefore, price mechanism plays an important role. Buyers want to choose spectrum that can accommodate required service with the lowest price while sellers wish to maximize their profits through selling (or leasing) the spectrum. Hence, the pricing mechanism in spectrum trading should be designed to offer profitable business to the sellers and create favorable services for the buyers. On the one hand, the spectrum price also depends on buyers' demands of spectrum and sellers' inventory of spectrum. When there are multiple buyers trying to bid for the same spectrum band, the price would rise. If multiple sellers can provide spectrum bands of similar service capacity, the price would fall. 
On the other hand, competition and cooperation among spectrum sellers and buyers in spectrum trading also significantly affect the pricing mechanism. A more liberalized spectrum market will spontaneously encourage competition and cooperation among different sellers and buyers. Competition allows spectrum sellers to survive and to make more profits by providing spectrum at a more reasonable price. Meanwhile, cooperation in both pricing and spectrum assignment among the sellers is helpful for their own benefits, considering the dynamics in spectrum spatio-temporal availability, user need, channel quality, and the potential multi-user diversity gain. For example, if the sellers cooperatively match high quality spectrum bands to the buyers requiring high quality bands, the overall spectrum efficiency is high, which makes it possible to accommodate more buyers and higher profits.

In [11], the monopolist-dominated quality-price contract is introduced as the pricing mechanism. It is offered by the spectrum seller and contains a set of quality-price combinations, each intended for a type of consumers. In [12], knapsack-based auction is employed to develop a dynamic pricing strategy for competitive spectrum sellers, which stimulates sellers to upgrade their network resources and offers better services for more profits. In [13], pricing dynamics in a competitive spectrum market consisting of selfish spectrum sellers are investigated. As a result of noncooperative nature of the sellers, price war occurs when the buyers are price-sensitive. In [14], an equilibrium pricing scheme is developed through a game-theoretic model for an environment in which multiple spectrum sellers compete mutually to offer buyers spectrum. Pricing mechanisms for spectrum trading with bandwidth uncertainty and spatial reuse are analyzed via game models in [15, 16]. In [17], the optimal investment and pricing decisions of a spectrum seller under spectrum supply uncertainty is investigated through a Stackelberg game.

If commodities in addition to spectrum bands, such as base stations (BSs) and power supply, are also involved in the spectrum trading, the adopted pricing mechanisms need to consider all such factors and generally become more complicated. A recent survey of pricing schemes in wireless environments can be found in [18], where pricing schemes are discussed and classified according to the factors involved in the price calculation, e.g., the available bandwidth and frequency of the spectrum, the negotiation capabilities between spectrum sellers and buyers, and the network facilities.

\subsection{Utility Functions}

Another critical issue in spectrum trading involves finding proper utility functions for optimizing resource allocation under practical constraints, such as bandwidth, power, data rate, and channel quality. Although price as discussed earlier is an important user consideration, user experience and satisfaction for a specific service need play an equally important role in resource allocation decision by spectrum trading.

Basically, utility functions map resource use (bandwidth, power, etc.) or performance criteria (data rate, delay, etc.) to their corresponding utility. The introduction of utility function allows its optimization as a metric for resource allocation. There are usually two approaches to obtaining or defining utility functions [19]. For a specific type of applications, the utility function can be obtained by sophisticated subjective surveys. For example, a utility function to indicate users' satisfaction toward throughput for best-effort services has been obtained in [20] through surveys. Another simpler and more popular method is to design utility functions based on engineers' quantified expectation of quality-of-service (QoS) with respect to objective indexes for delivered data streams, such as speed, accuracy, and latency, plus appropriate fairness in the network. The most commonly used utility functions of this category include throughput $[21,22]$, delay [23, 24], fairness [2528], net profit [29-31], energy efficiency [32-34], as well as sigmoid utility functions [35], etc.

Mathematical properties and tractability of utility functions are also crucial and need to be carefully considered when designing or choosing utility functions. For example, utility functions exhibiting convexity are generally easier to deal with than nonconvex ones. Moreover, in some game scenarios, utility functions satisfying certain conditions can guarantee network convergence and stability [36].

Another important issue on utility functions arises when optimizing the total utility of multiple entities. A common approach is to use the (weighted) sum or product of all individual utility functions as the overall utility function, where each individual utility function purely represents user's self-interest [37, 38]. This approach is reasonable when each individual is rational and committed to achieving the best outcome for itself regardless of impact on others. However, the rationality assumption about individuals is somewhat asocial and may not be adequate for truly cooperative scenarios. To well characterize the interplay among multiple entities in cooperative systems, satisficing theory [39, 40] and social utility functions [41, 42] are introduced as alternatives. Satisficing theory is used to explain the behavior of decision makers under circumstances in which an optimal solution cannot be determined or is not worth the efforts for pursuing it, considering that human beings can hardly evaluate all outcomes with sufficient precision or know the relevant probabilities of outcomes. It emphasizes that in many social scenarios 
the only information we can draw from for decisionmaking are the preferences of individuals. Social utility functions are constructed around multiple decisionmaker preferences rather than actions, thereby letting each individual expand one's sphere of interest beyond the self. Thus it can characterize complex interrelationship among individuals, such as cooperation, compromise, negotiation, and altruism.

\section{Models and Methods for Spectrum Trading}

In this section, we review some classical and stateof-the-art models and methods that can be applied to various spectrum trading scenarios, which are first briefly summarizes in Table 2, and then discussed in details.

\subsection{Conventional Optimization for Spectrum Trading}

A spectrum trading process can be simply regarded as an optimization problem, where spectrum sellers and/or buyers aim to maximize their respective utilities under certain constraints by finding out the optimal operating parameters [22, 34, 43-48]. Depending on spectrum trading scenario, various conventional optimization techniques, including linear programming, mixed integer linear programming, convex programming, mixed-integer convex programming, etc., may be applied to model and solve the problem.

There are two useful tricks for solving established optimization problems by classical optimization techniques. One is through transformation to simplify the problem. The other is through relaxation to provide better mathematical tractability. For example, when spectrum buyers maximize their throughput under interference constraints, the optimization problem can be established as a mixed-integer convex programming problem and then be solved by Lagrange dual decomposition [49] as in [22]. In [34], when the spectrum buyers optimize their energy efficiency, the problem can be set up as a mixed-integer nonconvex programming problem before being solved through a branch-andbound algorithm after relaxation and transformation.

\subsection{Game for Spectrum Trading}

Game is a mutually developed mathematical theory for understanding the strategic interplay among rational entities [50-52]. Because of underlying economic incentive structures in spectrum trading, it is quite natural to apply game theory there to model and analyze individual and group behaviors of entities involved, e.g., spectrum sellers and buyers, as widely discussed in some surveys [53-57] and more original contributions [15, 16, 25, 27, 58-73]. Using appropriate games to model various spectrum trading scenarios, the involved entities' strategic behaviors can be analyzed in formalized game structures. Moreover, game models can provide well defined equilibria and Pareto optimality/efficiency to characterize spectrum trading and may offer distributed solutions for noncentralized scenarios.

Mathematically, a game problem formulation consists of players, their strategies, and their payoff functions. A spectrum trading process can be modeled as a game, where the involved entities, e.g., spectrum sellers and buyers, are treated as the players, the feasible actions related to spectrum trading, e.g., spectrum to trade, transmission parameters, and pricing mechanisms, are treated as the strategies. The chosen utility functions, discussed in Section 3.2, are treated as payoff functions. When predicting outcomes of games, equilibria are an important family of solutions in which a unilateral deviation from the equilibrium strategy by one player would result in a lower payoff for the deviating player. Pareto optimality/efficiency is another important family of solutions in which it is impossible to make any player strictly better off without making at least one other player strictly worse off.

Depending on the availability of coordinators, spectrum trading can be modeled and analyzed as either non-cooperative spectrum trading game or cooperative spectrum trading game.

Non-Cooperative Spectrum Trading Game. In a noncooperative game [52], self-interest players have no enforceable cooperation mechanisms and make decisions independently. While players could cooperate, any cooperation must be self-enforcing [52]. If the strategies and payoff functions of the players are commonly known in a game ${ }^{1}$, it is a game of complete information; otherwise, it is called a game of incomplete information [52]. For a non-cooperative game of complete information, Nash equilibrium [51], if exists, is an important stable solution (to evaluate game outcome) in which no player can attain a higher payoff by unilaterally deviating his own strategy. Similarly, for a non-cooperative game of incomplete information, Bayesian Nash equilibrium [74], if exists, is an important stable solution. Because of the non-cooperative nature, non-cooperative games are suitable to model and solve the non-cooperative spectrum trading problems, which primarily focus on distributed design and cooperation stimulation. For example, the competitive behaviors of multiple spectrum sellers with complete information $[16,25]$ and with incomplete information $[15,62]$ as well as the competition among spectrum buyers [63] are

\footnotetext{
${ }^{1}$ This corresponds to the spectrum trading scenarios in which all network parameters including constraints, bandwidth, powers, channel gains, etc. are common knowledge to all those entities involved.
} 
Table. 2. Models and methods for spectrum trading

\begin{tabular}{c|l}
\hline Model & \multicolumn{1}{c}{ Method } \\
\hline Conventional optimization & $\begin{array}{l}\text { linear programming [45] } \\
\text { convex programming [43, 44, 46, 47] } \\
\text { nonconvex programming [22, 34] }\end{array}$ \\
\hline Game [53-57] & $\begin{array}{l}\text { noncooperative game: static [15, 16, 25, 62, 63], dynamic (repeated [65], multi-stage [64]), } \\
\text { Bayesian [66], sequential, Stackelberg [67, 68] }\end{array}$ \\
\hline Auction [79-81] & $\begin{array}{l}\text { cooperative game: coalition formation [69-71], Nash bargaining [27, 72, 73, 77] } \\
\text { single-unit [86] vs. multi-unit [87] } \\
\text { auction game: noncooperative [54, 58, 89] vs. cooperative [30] }\end{array}$ \\
\hline Graph & $\begin{array}{l}\text { conflict graph [90]: multi-point, dynamic [91] } \\
\text { factor graph [93] } \\
\text { bipartite graph [94-96] } \\
\text { layer-graph [97] }\end{array}$ \\
\hline
\end{tabular}

modeled as non-cooperative games and then analyzed through Nash equilibria.

In practice, some spectrum trading processes may last over such long periods that the spectrum spatiotemporal availability varies. An appropriate game model should enable its players to make timely decisions in respond to such network dynamics. In a static (non-cooperative) game (also namely strategic (noncooperative) game [52]), however, players make onetime decisions at the beginning of the game without change over time. On the other hand, a dynamic (non-cooperative) game (also namely extensive (noncooperative) game) allows players to make decisions not only at the beginning of the game but also at any time when they have to. If each player is perfectly informed of previous actions by all other players at each stage in the dynamic game, it is a game of perfect information; otherwise, it is a game of imperfect information. To properly model and analyze players' interplay evolving over time in dynamic spectrum trading, dynamic game models, such as repeated game, multi-stage game, Bayesian game, and sequential game [52], should be applied instead of static models. Depending on the availability of complete and perfect information, dynamic spectrum trading can be further divided into dynamic spectrum trading of complete information ${ }^{2}$ and dynamic spectrum trading of incomplete information.

- For dynamic spectrum trading scenarios of complete information, repeated game and multi-stage game

\footnotetext{
${ }^{2}$ In a slight abuse of terminology, spectrum trading of complete information refers to spectrum trading environments having complete and perfect information in the sense of game throughout this manuscript.
}

can be used as models. For these games, subgame perfect equilibrium [52] is widely used to predict the outcome. For example, the competition between two spectrum sellers [64] and the competitive behaviors of multiple selfish spectrum buyers [65] are modeled by a three-stage dynamic game and a repeated dynamic game, respectively, before analysis based on the subgame perfect equilibria.

- For dynamic spectrum trading scenarios of incomplete information, Bayesian game and sequential game, where players generate, and rely on, their self-renewing beliefs (i.e., probability distribution) over observation uncertainties regarding the actions of others, can be applied as models. For these games, perfect Bayesian equilibrium and sequential equilibrium [52] are commonly used to measure the outcome. For example, the competition for shared bandwidth among multiple spectrum buyers who are unable to completely observe other buyer behaviors (e.g., speed of movement, bandwidth demand) is modeled by a Bayesian game and subsequently measured by Bayesian Nash equilibrium in [66].

Another practical consideration in spectrum trading is that different entities may have different levels of priorities, e.g., some entities may be primary while others are subsidiary. Such spectrum trading scenarios can be well modeled by Stackelberg games. Stackelberg game [52] is a two-player extensive game with perfect information where a leader chooses an action from his strategy set and a follower, informed of the leader's choice, selects an action from its strategy set. The usual solution to such games is that of subgame perfect equilibrium. For example, a spectrum trading scenario with a single spectrum seller (aiming to maximize 
Table. 3. Game modeling and equilibria for non-cooperative spectrum trading.

\begin{tabular}{l|l|l}
\hline & Spectrum trading of complete information & Spectrum trading of incomplete information \\
\hline Static spectrum trading & $\begin{array}{l}\text { Static game of complete information } \\
\text { Nash equilibrium }\end{array}$ & $\begin{array}{l}\text { Static game of incomplete information } \\
\text { Bayesian Nash equilibrium }\end{array}$ \\
\hline Dynamic spectrum trading & $\begin{array}{l}\text { Dynamic game of complete information } \\
\text { Subgame perfect equilibrium }\end{array}$ & $\begin{array}{l}\text { Dynamic game of incomplete information } \\
\text { Perfect Bayesian equilibrium, sequential equilibrium }\end{array}$ \\
\hline
\end{tabular}

its revenue by properly adjusting its load across the spectrum) and multiple selfish spectrum buyers (aiming to maximize their own payoffs) is modeled as a Stackelberg game, in which the seller is treated as the leader while the buyers are viewed as the followers [67]. In [68], multiple legitimate parties (i.e., spectrum sellers), facing malicious eavesdroppers who also hold spectrum, allow multiple other terminals, (i.e., spectrum buyers), to rent spectrum if they help combat the malicious parties by acting as cooperative jammers. The spectrum trading process is modeled and analyzed as a Stackelberg game with legitimate parties as the leaders and friendly jammers as the followers.

In Table 3, we summarize the game modeling and equilibria for non-cooperative spectrum trading. Note that non-cooperative spectrum trading modeled by non-cooperative games may suffer low efficiency in spectrum utilization, a problem inherent to the equilibria in those games. As an alternative with possibly high spectrum utilization efficiency, cooperative spectrum trading also has attracted substantial attention.

Cooperative Spectrum Trading Game. A cooperative game [52] is a game, in which groups of players (also namely coalitions in game terminology) may enforce cooperative behaviors to maximize a common payoff of a coalition. Because of the cooperative nature, cooperative games can well model and solve the cooperative spectrum trading problems, in which cooperation among entities is enforced through centralized control. Coalition formation game and Nash bargaining game are two commonly used cooperative game models [52].

Coalition formation game [52] is a cooperative game admitting a set of players seeking to form coalitions to improve their payoffs. Classical coalitional problems are typically modeled in the characteristic form [75], in which the utility of a coalition is not affected by the formation of other distinct coalitions. In contrast, for coalitional games in partition form [76], the value of any coalition strongly depends on how other players outside the coalition are organized. The solution for coalition formation game is called the core [52], which is defined as the set of all undominated imputations [52]. Coalition formation game is adequate to model and analyze the spectrum trading where some involved entities want to cooperative to improve their transmission opportunities [69-71].
Nash bargaining game [52] can appropriately model and analyze the negotiation/bargaining processes of involved entities in the spectrum trading $[27,72,73$, 77]. And a Nash bargaining solution [52] is Pareto optimal for a Nash bargaining game.

\subsection{Auction for Spectrum Trading}

Auction theory [78], an applied branch of economics, deals with how entities act in auction markets and studies the properties of auction markets. In general, auction suits a situation where the commodity value to the buyers is uncertain and may even vary. For example, auction has been used historically by government authorities to sell licenses for some spectrum bands, such as bands for mobile communications. An auction is an event to trade/exchange commodities and may involve bidder(s), seller(s), and auctioneer(s) [78]. A bidder submits bids (i.e., bidding prices) to buy the commodities; a seller owns the commodities and offers asks (i.e., asking prices) to sell them; an auctioneer is an intermediate agent betweens bidders and sellers, and is in charge of the auction process.

Recently, auction theory has been recognized as a promising tool to efficiently solve various emerging spectrum trading problems, which has been extensively covered in some surveys and tutorials [79-81] as well as related literature [29, 30, 58, 82-89]. Spectrum trading can be modeled, analyzed, and implemented as auctions, where the spectrum buyers are bidders, the spectrum sellers remain as sellers, and the spectrum exchange or spectrum brokers or even the spectrum sellers act as auctioneers. Using auction models, we can analyze the outcome, efficiency, fairness, and optimal and equilibrium bidding and asking strategies of the spectrum trading.

Spectrum trading auctions can be categorized based on different criteria, including one-sided and two-sided spectrum trading auctions, single-unit and multi-unit spectrum trading auctions, and non-cooperative and cooperative spectrum trading auction games.

One-Sided and Two-Sided Spectrum Trading Auctions. Depending on the number of spectrum sellers and the number of spectrum buyers in the market, spectrum trading auctions can be categorized as one-sided 

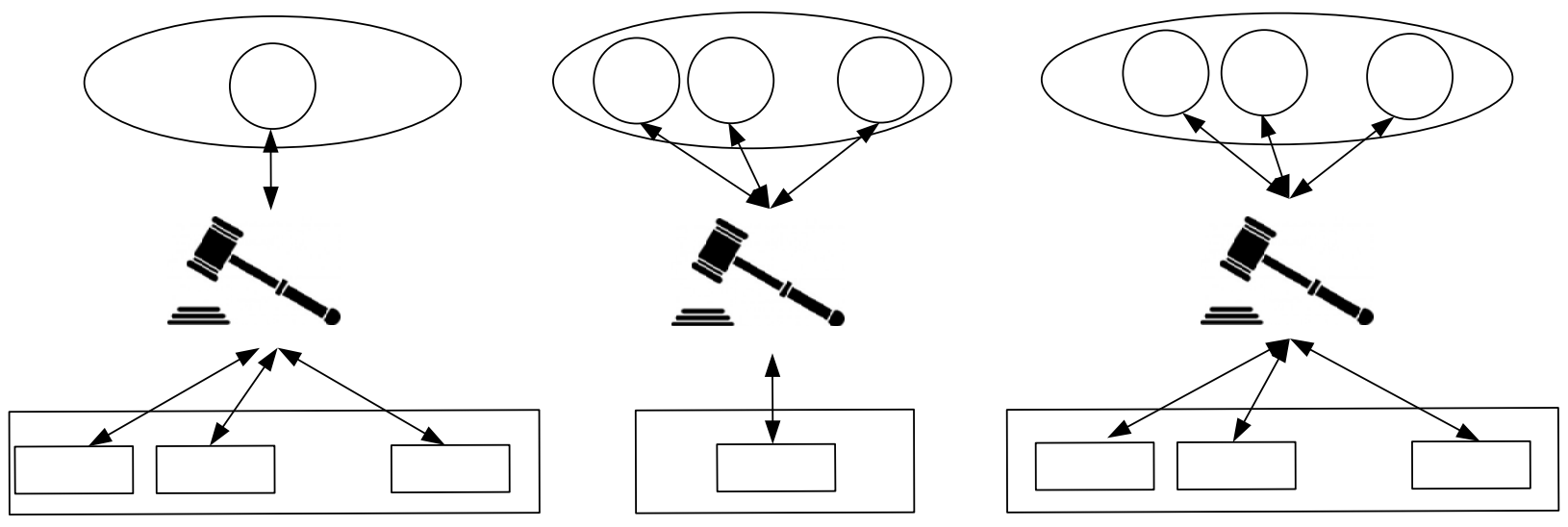

Figure 2. Spectrum trading auctions: (a) forward one-sided spectrum trading auction; (b) reverse one-sided spectrum trading; (c) two-sided spectrum trading auction.

spectrum trading auctions and two-sided spectrum trading auctions, as shown in Figure 2.

Usually, in a forward one-sided auction, the bidders submit their bids to a single seller; in a reverse onesided auction, the sellers offer their asks to a single bidder. Spectrum trading with a single spectrum seller and multiple spectrum buyers can be well modeled and analyzed as forward a one-sided auction while spectrum trading with a single spectrum buyer and multiple spectrum sellers can be properly modeled and analyzed as a reverse one-sided auction. For example, spectrum trading with a single spectrum seller and multiple spectrum buyers is modeled and analyzed as a forward one-sided auction in [82]. In [83], the spectrum trading process with multiple competing spectrum sellers is investigated as a reverse auction. VickreyClarke-Groves (VCG) auction [78] is used as a forward one-sided spectrum trading auction for competitive spectrum sellers to offer spectrum bands to buyers with and without contracts simultaneously in [29].

In a two-sided auction, bidders and sellers submit their bids and asks, respectively. The auctioneer then matches the asks and the bids before exchanging payment and commodity for each matching pair of bidder and seller. Spectrum trading with multiple spectrum sellers and multiple spectrum buyers can appropriately modeled and investigated as a twosided auction. For example, a general framework for truthful two-sided spectrum trading auctions for homogeneous spectrum bands is established for spectrum trading with multiple sellers and buyers in [84]. In [85], a truthful two-sided auction considering the heterogeneity of spectrum bands is developed for spectrum trading with multiple sellers and buyers.
Single-Unit and Multi-Unit Spectrum Trading Auctions. As a result of the dynamic nature of spectrum spatiotemporal availability, there could be multiple isolated unused spectrum bands available for spectrum trading. According to the number of spectrum bands that are auctioned at a time, spectrum trading auctions can be divided into single-unit spectrum trading auctions and multi-unit spectrum trading auctions.

In a single-unit auction [78], only one commodity is auctioned at a time. Spectrum trading with multiple spectrum bands for sale sequentially can be well modeled and analyzed as a single-unit spectrum trading auction as in [86].

In a multi-unit auction [78], multiple commodities are auctioned at a time. Spectrum trading with multiple spectrum bands for sale simultaneously can be appropriately modeled and investigated as a multi-unit spectrum trading auction shown in [87].

Non-Cooperative and Cooperative Spectrum Trading Auction Games. To model and analyze the strategic asking and bidding behaviors of spectrum sellers and buyers, and to enable decentralized design in spectrum trading auction, game theory can be naturally incorporated to form an auction game. In a spectrum trading auction game, participants' strategies are limited by the adopted auction machinery (i.e., the bidding/asking languages) while the auction outcome can be evaluated by equilibrium bidding and asking strategies. Depending on the level of cooperation among sellers and among bidders, spectrum trading auction games can be classified as either non-cooperative spectrum trading auction games or cooperative spectrum trading auction games.

For a one-sided spectrum trading auction game with non-cooperative bidders or non-cooperative sellers, Nash equilibrium is commonly viewed as the optimal 
outcome of the auction. For a two-sided spectrum trading auction game involving non-cooperative sellers and non-cooperative buyers, competitive equilibrium [54], a price at which the number of buyers willing to pay is equal to the number of sellers willing to accept, is a well defined theoretical prediction of the outcome. Moreover, considering spectrum trading auction over long periods, dynamic game can be employed to incorporate the spectrum dynamics and participants' bidding/asking history into the auction. The equilibria, such as perfect Bayesian equilibrium and sequential equilibrium or subgame perfect equilibrium, can be utilized to anticipate the outcome of dynamic spectrum trading auction games. For example, a double auction is established among multiple TV broadcasters (i.e., spectrum sellers) and WRAN service providers (i.e., spectrum buyers) for TV radio spectrum bands in [58]. The WRAN service providers compete with each other by adjusting the service price charged to WRAN users. A non-cooperative game is formulated to obtain the solution in terms of the number of TV bands and the service price of a service provider for maximum profit. In [89], a repeated auction game of incomplete information is established to characterize the strategic competitions among the multiple spectrum buyers for spectrum bands from a spectrum seller, where knowledge regarding other buyers is limited due to the distributed nature of the network.

In a cooperative spectrum trading auction environment, coalition formation game can be applied to maximize the sum expected payoff of the spectrum sellers or buyers in a coalition. The solution for the cooperative auction game is the core. For example, in [30] the interaction among the sellers in a secondary spectrum trading market is modeled as a cooperative auction game. It is further demonstrated that, for any fixed strategies of the buyers, the core of the cooperative auction game is nonempty.

\subsection{Graph for Spectrum Trading}

Graph theory can also be used for spectrum trading. To capture different features of different spectrum trading scenarios, various of graph structures can be utilized.

One of the most common approaches to spectrum trading is based on conflict graphs [90]. Conflict graphs can capture the interference relationship among the entities involved in spectrum trading. For example, each spectrum buyer is represented by one node. If two nodes interfere with each other, one edge is linked between them. Weights on edges can measure the interference levels, where different utility functions (e.g., those in Section 3.2) can be used to capture various requirements. When one node can be assigned to more than one spectrum bands, a multi-point conflict graph can be used. To capture the dynamics of interference, the spectrum trading system can be modeled by a dynamic conflict graph [91]. After the conflict graph is constructed, spectrum will be assigned accordingly. To avoid interference, connected nodes are normally assigned with different spectrum bands. To fulfill this goal, graph-coloring can be utilized [92], such that one color is used to represent one spectrum band and connected nodes may not have the same color. Besides using one node and one color to represent one spectrum buyer and one spectrum band, respectively, the conflict graph can be generalized to capture the spectrum trading system where a group of buyers share the same set of spectrum bands, with one node and one color representing a group of buyers and a set of spectrum bands, respectively. In spectrum trading, the interference information may be unknown or not accurately known to spectrum buyers (nodes). Factor graph [93] can model and tackle such spectrum trading scenarios using probabilistic inference approaches, such as belief propagation.

Another type of graph structures often used is the bipartite graph, where nodes representing buyers are put on one side whereas nodes representing spectrum bands are put on another side. This structure captures the actions between buyers and spectrum bands. An edge is connected between a buyer and a spectrum band if the buyer is a potential candidate for the spectrum. Similar to the conflict graph, weights can be put on edge to characterize the system requirements, such as throughput and energy efficiency. There are two known spectrum trading methods based on the bipartite graph, maximal matching and stable matching. Maximal matching methods, such as the Hungarian algorithm [94], lead to a maximal sum-weight spectrum trading result [95]. Unlike maximal matching methods, stable matching algorithms, such as the Gale-Shapley algorithm [94], take the preferences of nodes on both sides into account [96]. Nodes can build their preference lists according to their own requirements, not necessarily according to the same utility function.

Besides conflict graph, factor graph, and bipartite graph, other graph structures, such as layered graph [97] can also be used to analyze spectrum trading. A layered graph can capture the connection between spectrum assignment and routing path computation and may adequately model multi-hop spectrum trading environments.

\section{Spectrum Trading Based Dynamic Spectrum Sharing and Access}

In this section, we focus on spectrum trading based dynamic spectrum sharing and access in various wireless environments, including the highly popular cases of cognitive radio (CR), heterogeneous networks (HetNets), and device-to-device (D2D) transmissions. 


\subsection{Spectrum Trading for CR}

The scarcity of spectrum and the underutilization of most licensed spectrum bands have motivated the popularity of CR over the past decade [98-100]. CR may be one promising technology for spectrum efficient wireless access. A typical CR network often consists of three main entities: spectrum authorities, licensed users, and unlicensed users. The spectrum authorities normally refer to government agencies regulating spectral bands. Licensed users, also called primary users (PUs), hold licenses of spectrum bands issued by the spectrum authorities. PUs can be television stations, wireless telecom operators, and other related parties. Unlicensed users, also called secondary users (SUs), have lower priorities than the PUs but eagerly seek transmission opportunities on the licensed bands whenever possible. The concept of spectrum trading is apparently inherent to $\mathrm{CR}$, considering traffic dynamics and spectrum underutilization of PUs and transmission requirements of SUs. In other words, CR is simply one particular form of spectrum trading. Moreover, the secondary spectrum trading market model originates from the concept of CR. Next, we primarily focus on the spectrum trading activities among PUs and SUs in the secondary spectrum trading market $[2,3]$.

In the secondary spectrum trading market, PUs can sell or lease parts of their own bands either exclusively to the SUs or coexisting with them. Their objective is to maximize the utility of the licensed spectrum. In general, utility function of one PU will be an increasing function of its income from selling the bands while a decreasing function of its performance loss due to allowing SUs to use its bands. SUs want to buy or rent spectrum bands from PUs. Their goal is to minimize the cost while maximizing their payoff, such as throughput. The utility function of one SU will be an increasing function of its own performance while a decreasing function of its cost. Based on these fundamental observations, there exist a number of works on spectrum trading in $\mathrm{CR}$, including several survey [53-55, 57], pricing mechanisms [11, 14-17], utility functions [36], spectrum trading games [15, 16, $25,27,58,59,62,67]$, spectrum trading auctions [58, $82,89]$, graph-based spectrum trading [90, 91, 93, 96, 97], among others.

\subsection{Spectrum Trading for HetNets}

HetNets may consist of macro BSs and micro BSs, which typically transmit at high power levels, overlaid with several pico BSs, femto BSs, and relay BSs, that transmit at substantially lower power levels [101] for local coverage. The high-power BSs are in charge of the general coverage area while the low-power BSs can be deployed to eliminate coverage holes in the macroonly systems and to improve quality and throughput in high activity (hot) spots. However, to suppress interference among different BSs, the current spectrum sharing policy in HetNets is static or inflexible, which may sacrifice the full capacity of spectrum. To tackle this issue, spectrum trading has also been introduced into HetNets for more flexible and dynamic spectrum sharing [71, 77, 102-105].

In [71], a framework for macrocell-femtocell cooperation under a closed access policy is proposed, where a femtocell user may act as a relay for macrocell users and is rewarded with a fraction of the frame duration used by macrocell users for transmission. A coalitional formation game is used to model and analyze the spectrum trading process and is solved by the recursive core of the game. The data rates for both macrocell and femtocell users can be greatly improved.

In a two-tier HetNet, spectrum trading, in which small cells may dynamically open portion of the access opportunities to macrocells for profits, is investigated as a hierarchical dynamic game [102]. At the lower level, an evolutionary game is formulated to model and analyze the adaptive service selection of users. At the upper level, a Stackelberg game, in which macrocells are leaders while small cells are followers, is formulated to characterize the pricing strategy of macrocells and the open access ratio strategy of small cells. The resulting dynamic control outperforms its static counterpart.

In [103], a femtocell expects to rent spectrum from a coexisting macrocell to serve its end users and also allows hybrid access of the macrocell users to improve utilities of both femtocell and macrocell. The whole spectrum trading procedure is modeled and analyzed as a three-stage Stackelberg game, in which the macrocell and the femtocell determine the spectrum leasing ratio, spectrum leasing price, and open access ratio sequentially. It is shown that both tiers can benefit from the spectrum leasing. In fact, the hybrid access of femtocell can further improve their utilities.

\subsection{Spectrum Trading for D2D}

In cellular networks, nearby mobile terminals may communicate directly without going through the base station (as a relay). Such direct terminal-to-terminal link is known as D2D communications [95]. D2D communication as an underlaying network to cellular networks can share the use of cellular resources for better spectral utilization so long as its interference to co-channel cellular users is appropriately contained. Because of the underlaying nature of D2D transmission, a similar spectrum trading scenario as in a secondary spectrum trading in CR arises from cellular networks to support underlying D2D mechanisms.

In [43], a spectrum trading problem of D2D users and prioritized cellular users is investigated. It is demonstrated that optimized spectrum trading 
improves the total throughput without generating harmful interference to cellular networks.

A spectrum trading environment where D2D users seek transmission opportunities when sharing spectrum with uplink cellular users while guaranteeing the QoS of normal cellular users is studied in [44]. Despite the nonconvexity of the optimization problem, an analytical characterization of the globally optimal non-orthogonal spectrum sharing strategy is given.

In [60], a spectrum trading scenario focusing on mode selection for energy-efficient D2D communications is modeled as a coalitional game. Stable coalitions, where no D2D link can change its communication mode to lower transmission cost without making others worse off, are found as optimal solutions.

\section{Open Issues}

In the previous sections, we have provided a comprehensive overview of spectrum trading that can significantly improve spectrum efficiency. However, there are still some open issues on the development of more flexible and more efficient spectrum trading.

\subsection{Novel Modeling of Spectrum Trading Market}

The development of spectrum markets for wireless service provision requires an evolving spectrum trading market model. This model should consider the latest advancement in wireless technology and be adequately supported by the latest wireless technological infrastructure. For example, in HetNets terminals from different cells (i.e., different spectrum buyers) may operate on the same band. The current spectrum trading market models seldom consider the trading of a spectrum to multiple spectrum buyers simultaneously or say the coexistence of multiple spectrum buyers within the same band. How to manage such coexistence needs to be well incorporated in new spectrum trading market models.

\subsection{Spectrum Trading for Emergency Response}

Under severe conditions or extreme cases, e.g., major disasters (e.g. earthquake and hurricane), first responders need to locate and rescue trapped survivors and maintain reliable communications between responders and public safety agencies at a time when regular civilian communications may be jammed or be out of service. The infrastructure of currently existing wireless communication systems is inadequate to fully meet the future demands of such emergency responses from the perspectives of security, reliability, and robustness. Contrarily, trading based spectrum paradigm potentially can realize efficient and reliable emergency network transmission, by offloading and redistributing regular non-urgent traffics to spare or low quality spectrum bands while establishing at the same time a coordinated inter-agency rapid response network. However, how to use spectrum trading to develop interoperable communications system that would integrate existing radio frequency infrastructures for public services, including fire, police and emergency medical services, hospitals, emergency rooms and trauma centers, with an Internet Protocol (IP) backbone while maintaining connectivity for mobile terminals remains to be another challenge.

\subsection{Spectrum Trading for Social Benefit Network}

Social benefit networks, which cost users less than regular commercial one, can be set up by opportunistically exploiting the spectrum vacancies, without the necessity of strictly ensuring a certain level of QoS. For example, when the high-rate spectrum bands are lightlyloaded with regular users, low-income users may enjoy high-rate services for skill training and commerce at a deep discount; when the high-rate spectrum bands become crowded with regular user, low-income users can be handed over to other low-rate spectrum bands without considering its QoS. How to schedule the overall traffic and adjust the resources allocated to socially or economically disadvantaged users to balance the social and economic values of spectrum is an interesting challenge.

\subsection{Transmission across Broad Multi-Spectrum Bands}

Given highly dynamic user requirements and spectrum availability, resource allocation in flexible spectrum trading based systems is less likely to assign contiguous spectrum to a single user whose service request often consists of multiple data flows of varying QoS needs. First, acquisition of contiguous broad spectrum is more difficult and costly. In fact, costs at different wavelengths often differ as some bands are more suitable for mobile users while others are more suitable for high-rate low mobility users. Second, different user data flows may demand different QoS levels that are not monolithic. The cost can be substantially reduced when users are assigned fragmented, multi-spectrum resources to match their data QoS needs. Another important reason for fragmented multispectrum resource allocation lies in the broad bandwidth ranging from the lower very high frequency (VHF) $(54-88 \mathrm{MHz}, 174-216 \mathrm{MHz})$ once vacated by terrestrial TV stations up to millimeter wave V-band $(60 \mathrm{GHz})$. These bands are inherently fragmented with multiple special purpose licensees. 


\subsection{Channel Acquisition across Broad Multi-Spectrum Bands}

In flexible spectrum trading based systems, broadband channel acquisition/estimation is essential for achieving multi-spectrum diversity. Spectrum sellers that dynamically allocate their users across broad multispectrum bands plus mobile users flexibly switching among multi-spectrum bands in a proactive manner will result in a higher spectral efficiency and/or better QoS. Normally, pilots and channel estimation for a single-band are specifically designed according to prior historic information on channel characteristics. For example, more pilots are required for a highfrequency band to account for faster fading and longer delay-spread channel states whereas fewer pilots are deployed for a low-frequency band that tends to exhibit slower fading and shorter delay spread. However, to acquire/estimate (instantaneous) channel state information (CSI) of different channels across broad multispectrum bands simultaneously is much more challenging.

\subsection{Broadband Sensing}

Till now, there has only been limited works on wideband sensing for CR networks [106-108]. To adapt to various applications in free spectrum trading, system feature should be taken into account. In order to reduce wideband processing time, service searching procedure must be shortened. Without negotiating with many spectrum sellers at the same time, it is more efficient for each spectrum seller to pronounce a range about their service quality and the corresponding charge. With such information, users can choose among a small number of spectrum sellers based on the coarse information and perform additional price and service negotiating processes with only a small number of remaining spectrum sellers. Only when the coarse service searching is done across a broadband spectrum, will the ensuing procedure proceed on a set of given spectrum bands. This process can reduce complexity. In brief, different features need to be taken into account to make the system practical.

\section{Conclusions}

Because of the non-uniform need and heterogeneity of radio wireless communications temporally and spatially, traditional rigid spectrum management relying on centralized command and control tends to be inefficient. In fact, such centralized approach has resulted in massive underutilization of some spectrum bands while overcrowding others. Spectrum trading can improve social and economic values of the spectrum by taking advantage of market-based mechanisms and thereby becomes a promising spectrum management approach in wireless communications. This article presents an overview of spectrum trading, ranging from the fundamental structure of spectrum trading markets to the state-of-the-art techniques for modeling and solving various spectrum trading problems, including spectrum trading based dynamic spectrum sharing and access. Furthermore, we identify some open challenges in spectrum trading for future research in this area.

\section{References}

[1] FCC (2002) Spectrum Policy Task Force Report. Tech. rep., FCC ET Docket 02-155.

[2] FCC (2003) The development of secondary markets-Report and Order and further notice of proposed rule marketing. Tech. rep., FCC 03-113.

[3] FCC (2004) Second report and order: Promoting efficient use of spectrum through elimination of barrier to the development of secondary markets. Tech. rep., FCC 04167.

[4] FCC (2010) Unlicensed Operations in the TV Broadcast Bands, Second Memorandum Opinion and Order. Tech. rep., FCC 10-174.

[5] Caicedo, C.E. and Weiss, M.B.H. (2011) The viability of spectrum trading markets. IEEE Commun. Mag. 49(3): 46-62.

[6] Berry, R., Honig, M. and Vohra, R. (2010) Spectrum markets: Motivation, challenges and implications. IEEE Commun. Mag. 48(11): 146-155.

[7] Caicedo, C.E. and Weiss, M. (2008) An analysis of market structures and implementation architectures for spectrum trading markets. In Telecommun. Policy Research Conf. (Fairfax, VA).

[8] Berry, R. (2012) Network market design part II: Spectrum markets. IEEE Commun. Mag. 50(11): 84-90.

[9] Tonmukayakul, A. and Weiss, M. (2008) A study of secondary spectrum use using agent-based computational economics. Netnomics 9(2): 125-151.

[10] NoAm, E. (2012) The economists' contribution to radio spectrum access: The past, the present, and the future. IEEE Proc. 100(Special Centennial Issue): 1692-1697.

[11] GAO, L., WANG, X.B., XU, Y.Y. and Zhang, Q. (2011) Spectrum trading in cognitive radio networks: $\mathrm{A}$ contract-theoretic modeling approach. IEEE. J. Sel. Areas Commun. 29(4): 843-855.

[12] Sengupta, S. and Chatterjee, M. (2009) An economic framework for dynamic spectrum access and service pricing. IEEE/ACM Trans. Netw. 17(4): 1200-1213.

[13] Xing, Y., Chandramouli, R. and Cordeiro, C.M. (2007) Price dynamics in competitive agile spectrum access markets. IEEE J. Sel. Areas Commun. 25(3): 613-621.

[14] Niyato, D. and Hossain, E. (2008) Competitive pricing for spectrum sharing in cognitive radio networks: Dynamic game, inefficiency of Nash equilibrium, and collusion. IEEE J. Sel. Areas Commun. 26(1): 192-202.

[15] Kasbekar, G. and Sarkar, S. (2010) Spectrum pricing games with bandwidth uncertainty and spatial reuse in cognitive radio networks. Proc. ACM Int. Symp. Mobile Ad Hoc Netw E Comput. (MobiHoc'10). 
[16] Kasbekar, G.S. and SARKar, S. (2012) Spectrum pricing games with spatial reuse in cognitive radio networks. IEEE J. Sel. Areas Commun. 30(1): 153-164.

[17] Duan, L., Huang, J. and Shou, B. (2011) Investment and pricing with spectrum uncertainty: A cognitive operators perspective. IEEE Trans. Mobile Comput. 10(11): 1590-1604.

[18] Gizelis, C.A. and Vergados, D.D. (2011) A survey of pricing schemes in wireless networks. IEEE Commun. Surveys Tutorials 13(1): 126-145.

[19] SonG, G.C. and LI, G.Y. (2005) Utility-based resource allocation and scheudling in OFDM-based wireless broadband networks. IEEE Commun. Mag. : 127-134.

[20] JiAnG, Z., GE, Y. and Li, G.Y. (2005) Max-utility wireless resource management for best-effort traffic. IEEE Trans. Wireless Commun. 04(1): 100-111.

[21] Eraslan, B., GozupeK, D. and Alagoz, F. (2011) An acution theory based algorithm for throughput maximizing scheduling in centralized cognitive raido networks. IEEE Commun. Lett. 15(7): 734-736.

[22] Zhou, X., Li, G., Li, D., WANG, D. and Soong, A. (2010) Probabilistic resource allocation for opportunistic spectrum access. IEEE Trans. Wireless Commun. 9(9): 2870-2879.

[23] CuI, Y., LAu, V.K.N. and R. WANG, H.H. (2012) A survey on delay-aware resource control for wireless systemsLarge deviation theory, stochastic lyapunov drift, and distributed stochastic learning. IEEE Trans. Info. 58(3): 1677-1701.

[24] Shi, X., Medard, M., and Lucani, D. E. (2013) Spectrum assignment and sharing for delay minimization in multi-hop multi-flow CRNs. IEEE J. Sel. Areas Commun. 31(11): 2483-2493.

[25] Niyato, D., Hossain, E. and Han, Z. (2009) Dynamics of multiple-seller and multiple-buyer spectrum trading in cognitive radio networks: A game-theoretic modeling approach. IEEE Trans. Mobile Comput. 8(8): 1009-1022.

[26] Zheng, L. and TAN, C.W. (2013) Cognitve raido network duality and algorithms for utlity maximization. IEEE J. Sel. Areas Commun. 31(3): 500-513.

[27] Ni, Q. and Zarakovitis, C.C. (2012) Nash bargaining game theoretic scheduling for joint channel and power allocation in cognitive radio systems. IEEE J. Sel. Areas Commun. 30(1): 70-71.

[28] Bian, K., Park, J.M., Du, X. and LI, X. (2013) Enabling fair spectrum sharing: Mitigating selfish misbehaviors in spectrum contention. IEEE Netw. 27(3): 16-21.

[29] Gao, L., Huang, J., Chen, Y.J. and Shou, B. (2013) An integrated contract and auction design for secondary spectrum trading. IEEE J. Sel. Areas Commun. 31(3): 581-592.

[30] Chun, S.H. and LA, R.J. (2013) Secondary spectrum trading-auction-based framework for spectrum allocation and profit sharing. IEEE/ACM Trans. Netw. 21(1): $176-189$.

[31] Xu, D., Liu, X. and Han, Z. (2013) Decentralized bargain: A two-tier market for efficient and flexible dynamic spectrum access. IEEE Trans. Mobile Comput. 12(9): 1697-1711.

[32] Li, G., Xu, Z., Xiong, C., Yang, C., Zhang, S., Chen, Y. and Xu, S. (2011) Energy-efficient wireless communications: Tutorial, survey, and open issues. IEEE Wireless Commun. 18(6): 28-35.

[33] LI, L., Zhou, X., Xu, H., LI, Y.G., WAng, D. and Soong, A. (2010) Energy-efficient transmission in cognitive radio networks. In Proc. IEEE Consumer Commun. Netw. Conf. (CCNC'10).

[34] Xiong, C., Lu, L. and Li, G.Y. (2014) Energy-efficient spectrum access in cognitive radios. IEEE J. Sel. Areas. Commun. 32(3).

[35] Kuo, W.H. and Liao, W. (2008) Utility-based radio resource allocation for QoS traffic in wireless networks. IEEE Trans. Wireless Commun. 7(7): 2714-2722.

[36] Zhao, Y., Mao, S., Neel, J.O. and Reed, J.H. (2009) Performance evaluation of cognitive radios: Metrics, utility functions, and methodology. IEEE Proc. 97(4): 642-659.

[37] Song, G.C. and Li, G.Y. (2005) Cross-layer optimization for OFDM wireless networks-part I: Theoretical framework. IEEE Trans. Wireless Commun. 4(2): 614624.

[38] Song, G.C. and LI, G.Y. (2005) Cross-layer optimization for OFDM wireless networks-part II: Algorithm development. IEEE Trans. Wireless Commun. 4(2): 625-634.

[39] Simon, H. (1957) A behavioral model of rational choices. In Models of Man, social and rational: mathematical essays on rational human behavior in a social setting (New York: Wiley).

[40] Stirling, W.C. (2003) Satisficing Games and Decision Making: With Applications to Engineering and Computer Science (Cambridge University Press).

[41] Stirling, W.C. (2005) Social utility functions-part I: Theory. IEEE Trans. Sys. Man Cybernetics-Part C: Applications \& Reviews 35(4): 522-532.

[42] Stirling, W.C. and Frost, R.L. (2005) Social utility functions-part I: Applications. IEEE Trans. Sys. Man Cybernetics-Part C: Applications \& Reviews 35(4): 533543.

[43] Yu, C.H., Doppler, K., Ribeiro, C. and Tirkkonen, O. (2011) Resource sharing optimization for device-todevice communication underlaying cellular networks. IEEE Trans. Wireless Commun. 10(8): 2752-2763.

[44] Wang, J., Zhu, D., Zhao, C., Li, J. and Lei, M. (2013) Resource sharing of underlaying device-to-device and uplink cellular communications. IEEE Commun. Lett. 17(6): 1148-1151.

[45] Hoang, A.T. and Liang, Y.C. (2008) Downlink channel assignment and power control for cognitive radio networks. IEEE Trans. Wireless Commun. 7(8): 31063117.

[46] Pei, Y. and Liang, Y.C. (2013) Resource allocation for device-to-device communications overlaying two-way cellular networks. IEEE Trans. Wireless Commun. 12(7): 3611-3621.

[47] Zhang, R. and Liang, Y.C. (2008) Exploiting multiantennas for opportunistic spectrum sharing in cognitive radio networks. IEEE J. Sel. Areas Commun. 2(1): 88-102.

[48] Zhai, C., Zhang, W. and Ching, P. (2013) Cooperative spectrum sharing based on two-path successive relaying. IEEE Trans. Commun. 61(6): 2260-2270. 
[49] Boyd, S. and Vandenberghe, L. (2004) Convex Optimization (Cambridge University Press).

[50] Neumann, J.V. and Morgenstern, O. (1944) Theory of Games and Economic Behavior (Princeton University Press).

[51] Nash, J. (1950) Non-cooperative games. Ann. Math. 54(2): 286-295.

[52] Osborne, M.J. and Rubinstein, A. (1994) A Course in Game Theory (The MIT Press).

[53] Zhao, Q. and Sadler, B.M. (2007) A survey of dynamic spectrum access. IEEE Signal Process. Mag. 24(3): 79-89.

[54] JI, Z. and LiU, K.J.R. (2007) Cognitive radios for dynamic spectrum access - Dynamic spectrum sharing: A game theoretical overview. IEEE Commun. Mag. 45(5): 88-94.

[55] Niyato, D. and Hossain, E. (2008) Spectrum trading in cognitive radio networks: A market-equilibrium-based approach. IEEE Wireless Commun. 15(12): 71-80.

[56] Akkarajitsakul, K., Hossain, E., Niyato, D. and Kim, D.I. (2011) Game theoretic approaches for multiple access in wireless networks: A survey. IEEE Commun. Surveys E Tutorials 13(3): 372-395.

[57] WANG, B., Wu, Y. and LiU, K. J. (2010) Game theory for cognitive radio networks: An overview. Computer Networks, 54(14): 2537-2561.

[58] Niyato, D., Hossain, E. and Han, Z. (2009) Dynamic spectrum access in IEEE 802.22-based cognitive wireless networks: A game theoretic model for competitive spectrum bidding and pricing. IEEE Wireless Comm. 16(2): 16-23.

[59] Kasbekar, G. and Sarkar, S. (2011) Spectrum pricing games with arbitrary bandwidth availability probabilities. In Proc. IEEE Int. Symp. Inf. Theory (ISIT'11).

[60] Akkarajitsakul, K., Phunchongharn, P. and Hossain, E. (2012) Mode selection for energy-efficient D2D communications in LTE-advanced networks: A coalitional game approach. In Proc. IEEE Int. Conf. Commun. Sys. (ICCS'12) (Singapore).

[61] Wang, F., Song, L., Han, Z., ZhaO, Q. and Wang, X. (2013) Joint scheduling and resource allocation for device-to-device underlay communications. In IEEE Wireless Commun. E Netw. Conf. (WCNC'13) (Shanghai, China).

[62] Kasbekar, G.S. and Sarkar, S. (2012) Spectrum pricing games with random valuations of secondary users. IEEE J. Sel. Areas Commun. 30(11): 2262-2273.

[63] Han, Z., Ji, Z. and Liu, K.J.R. (2007) Non-cooperative resource competition game by virtual referee in multicell OFMDA networks. IEEE J. Sel. Areas Commun. 25(6): 1079-1090.

[64] Duan, L., Huang, J. and Shou, B. (2012) Duopoly competition in dynamic spectrum leasing and pricing. IEEE Trans. Mobile Comput. 11(11): 1706-1719.

[65] Etkin, R., Parekh, A. and Tse, D. (2007) Spectrum sharing for unlicensed bands. IEEE J. Sel. Areas Commun. 25(3): 517-528.

[66] Akkarajitsakul, K., Hossain, E. and Niyato, D. (2011) Distributed resource allocation in wireless networks under uncertainty and application of Bayesian game. IEEE Commmun. Mag. 49(8): 120-127.
[67] Haddad, M., Elayoubi, S.E., Altman, E. and Altman, Z. (2011) A hybrid approach for radio resource management in heterogeneous cognitive networks. IEEE J. Sel. Areas Commun. 29(4): 831-842.

[68] Stanojev, I. and Yener, A. (2013) Improving secrecy rate via spectrum leasing for friendly jamming. IEEE Trans. Wireless Commun. 12(1): 134-145.

[69] SaAd, W., Han, Z., Debbah, M. and Hjorungnes, A. (2009) A distributed coalition formation framework for fair user cooperation in wireless networks. IEEE Trans. Wireless Commun. 8(9): 4580-4593.

[70] SaAd, W., Han, Z., Debbah, M., Başar, T. and Hjørungnes, A. (2009) Coalitional game theory for communication networks. IEEE Signal Process. Mag. 26(6): 77-97.

[71] Pantisano, F., Bennis, M., Saad, W. and Debbah, M. (2012) Spectrum leasing as an incentive towards uplink macrocell and femtocell cooperation. IEEE J. Sel. Areas Commun. 30(3): 617-630.

[72] HAN, Z., Ji, Z. and LiU, K. (2005) Fair multiuser channel allocation for OFDMA networks using Nash bargaining solutions and coalitions. IEEE Trans. Commun. 53(8): 1366-1376.

[73] Zhang, Z., Shi, J., Chen, H.H., Guizani, M. and QIU, P. (2008) A cooperation strategy based on Nash bargaining solution in cooperative relay networks. IEEE Trans. Veh. Techonol. 57(4): 2570-2577.

[74] Harsanyi, J.C. (1967) Games with incomplete information played by "Bayesian" players, I-III: Part I. The basic model. Management Science 14(159-182).

[75] Ray, D. (2007) A Game-Theoretic Perspective on Coalition Formation (New York: USA: Oxford University Press).

[76] Han, Z., Niyato, D., SAAd, W., Başar, T. and HJørungnes, A. (2011) Game theory in wireless and communications networks: Theory, models and applications (Cambridge, UK: Cambridge Univeristy Press).

[77] Lin, P., Zhang, J., Zhang, Q. and Hamdi, M. (2013) Enabling the femtocells: A cooperation framework for mobile and fixed-line operators. IEEE Trans. Wireless Commun. 12(1): 158-167.

[78] Klemperer, P. (2004) Auctions: Theory and Practice (Princeton Univeristy Press).

[79] Zhang, Y., Lee, C., Niyato, D. and Wang, P. (2013) Auction approaches for resource allocation in wireless systems: A survey. IEEE Commun. Surveys \& Tutorials 15(3): 1020-1041.

[80] Zhang, Y., Niyato, D., Wang, P. and Hossain, E. (2012) Auction-based resource allocation in cognitive radio systems. IEEE Commun. Mag. 50(11): 108-120.

[81] Iosifidis, G. and Koutsopoulos, I. (2011) Challenges in auction theory driven spectrum management. IEEE Commun. Mag. 49(8): 128-135.

[82] WANG, X., Li, Z., Xu, P., XU, Y., GaO, X. and Chen, H.H. (2010) Spectrum sharing in cognitive radio networksAn auction-based approach. IEEE Trans. Sys. Man Cybernetics, Part B: Cybernetics 40(3): 587-596.

[83] GaO, L., Xu, Y. and WANG, X. (2011) MAP: Multiauctioneer progressive auction in dynamic spectrum access. IEEE Trans. Mobile Comput. (99).

[84] Zhou, X. and Zheng, H. (2009) TRUST: A general framework for truthful double spectrum auctions. 
In Proc. IEEE Int. Conf. Computer Commun. (INFOCOM'09).

[85] Feng, X., Chen, Y., Zhang, J., Zhang, Q. and Li, B. (2012) TAHES: A truthful double auction mechanism for heterogeneous spectrums. Wireless Communications, IEEE Transactions on 11(11): 4038-4047.

[86] Bae, J., Beigman, E., Berry, R.A., Honig, M.L. and Vohra, R. (2008) Sequential bandwidth and power auctions for distributed spectrum sharing. IEEE J. Sel. Areas Commun. 26(7): 1193-1203.

[87] Chen, Z., Huang, H., Sun, Y.e. and Huang, L. (2013) True-MCSA: A framework for truthful double multi-channel spectrum auctions. IEEE Trans. Wireless Commun. 12(8): 3838-3850.

[88] Sun, Y., Jover, R. and WANG, X. (2012) Uplink interference mitigation for OFDMA femtocell networks. IEEE Trans. Wireless Commun. 11(2): 614-625.

[89] Han, Z., Zheng, R. and Poor, H.V. (2011) Repeated auctions with Bayesian nonparametric learning for spectrum access in cognitive radio networks. IEEE Trans. Wireless Commun. 10(3): 890-900.

[90] Plummer, A. and Biswas, S. (2011) Distributed spectrum assignment for cognitive networks with heterogeneous spectrum opportunities. Wireless Commun. and Mobile Computing 11(9): 1239-1253.

[91] Hoang, A. and Liang, Y. (2008) Downlink channel assignment and power control for cognitive radio networks. IEEE Trans. Wireless Commun. 7(8): 31063117.

[92] Peng, C., Zheng, H. and Zhao, B.Y. (2006) Utilization and fairness in spectrum assignment for opportunistic spectrum access. Mobile Networks and Applications 11(4): 555-576.

[93] Chen, S., Huang, Y. and Namuduri, K. (2011) A factor graph based dynamic spectrum allocation approach for cognitive network. In Proc. IEEE Wireless Commun. Netw. Conf.: 850-855.

[94] West, D.B. (2001) Introduction to Graph Theory (Prentice Hall), 2nd ed.

[95] Feng, D., Lu, L., Yuan-Wu, Y., Li, G.Y., Feng, G. and LI, S. (2013) Device-to-device communications underlaying cellular networks. IEEE Trans. Commun. 61(8): 3541-3551.

[96] Lu, L., He, D., Yu, X. and Li, G.Y. (2013) Energy-efficient resource allocation for cognitive radio networks. In Proc. IEEE Global Commun. Conf.
[97] XIN, C., MA, L. and SHen, C.C. (2008) A path-centric channel assignment framework for cognitive radio wireless networks. Mobile Networks and Applications 13(5): 463-476.

[98] Mitola, J. and Maguire, G.Q. (1999) Cognitive radio: Making software radios more personal. IEEE Pers. Commun. 6(4): 13-18.

[99] MA, J., Li, G.Y. and JuAng, B.H. (2009) Signal processing in cognitive radio. Proc. IEEE 97(5): 805-823.

[100] Lu, L., Zhou, X., Onunkwo, U. and Li, G.Y. (2012) Ten years of research in spectrum sensing and sharing in cognitive radio. EURASIP J. Wireless Commun. and Netw. 2012.

[101] Damnjanovic, A., Montojo, J., Wei, Y., Ji, T., Luo, T., Vajapeyam, M., Yoo, T. et al. (2011) A survey on 3GPP heterogeneous networks. IEEE Wireless Commun. 18(3): 10-21.

[102] Zhu, K., Hossain, E. and Niyato, D. Pricing, spectrum sharing, and service selection in two-tier small cell networks: A hierarchical dynamic game approach. to appear in IEEE Trans. Mobile Comput. .

[103] YI, Y., Zhang, J., Zhang, Q. and Jiang, T. (2012) Spectrum leasing to femto service provider with hybrid access. In Proc. IEEE Int. Conf. Computer Commun. (INFOCOM'12).

[104] Cheng, S.M., Lien, S.Y., Chu, F.S. and Chen, K.C. (2011) On exploiting cognitive radio to mitigate interference in macro/femto heterogeneous networks. IEEE Wireless Commun. 18(3): 40-47.

[105] Gharehshiran, O., Attar, A. and Krishnamurthy, V. (2013) Collaborative sub-channel allocation in cognitive LTE femto-cells: A cooperative game-theoretic approach. IEEE Trans. Commun. 61(1): 325-334.

[106] Quan, Z., Cui, S., Sayed, A.H. and Poor, H.V. (2009) Optimal multiband joint detection for spectrum sensing in cognitive radio networks. IEEE Trans. Signal Process. 57: 1128-1140.

[107] Paysarvi-Hoseini, P. and Beaulieu, N.C. (2011) Optimal wideband spectrum sensing framework for cognitive radio systems. IEEE Trans. Signal Process. 59: 11701182.

[108] Sun, H., Nallanathan, A., Wang, C.X. and Chen, Y. (2013) Wideband spectrum sensing for cognitive radio networks: A survey. IEEE Wireless Commun. 20(2): 7481. 